Article

\title{
Magnetic Field Sensing Based on Magnetic-Fluid-Clad Multimode-Singlemode-Multimode Fiber Structures
}

\author{
Jiali Tang, Shengli Pu *, Shaohua Dong and Longfeng Luo \\ College of Science, University of Shanghai for Science and Technology, Shanghai 200093, China; \\ E-Mails: tangjiali1230@gmail.com (J.T.); zhuiguang0903@163.com (S.D.); \\ longfengllf@163.com (L.L.) \\ * Author to whom correspondence should be addressed; E-Mail: shlpu@usst.edu.cn; \\ Tel.: +86-21-6566-6454; Fax: +86-21-6566-7144.
}

External Editor: Vittorio M.N. Passaro

Received: 17 July 2014; in revised form: 22 September 2014 / Accepted: 8 October 2014 /

Published: 14 October 2014

\begin{abstract}
Magnetic field sensing based on magnetic-fluid-clad multimode-singlemodemultimode fiber structures is proposed and experimentalized. The structures are fabricated out using fiber fusion splicing techniques. The sensing principle is based on the interference between the core mode and cladding modes. Two interference dips are observed in our spectral range. Experimental results indicate that the magnetic field sensing sensitivities of $215 \mathrm{pm} / \mathrm{mT}$ and $0.5742 \mathrm{~dB} / \mathrm{mT}$ are obtained for interference dip around $1595 \mathrm{~nm}$. For interference dip around $1565 \mathrm{~nm}$, the sensitivities are $60.5 \mathrm{pm} / \mathrm{mT}$ and $0.4821 \mathrm{~dB} / \mathrm{mT}$. The response of temperature is also investigated. The temperature sensitivity for the dip around $1595 \mathrm{~nm}$ is obtained to be $9.93 \mathrm{pm} /{ }^{\circ} \mathrm{C}$.
\end{abstract}

Keywords: magnetic fluid; multimode-singlemode-multimode fiber structure; mode interference

\section{Introduction}

Magnetic fluid (MF) is a kind of stable colloidal suspension system consisting of surfactant-coated magnetic nanoparticles with typical sizes of $10 \mathrm{~nm}$ dispersed in a suitable liquid carrier. It possesses both the features of magnetic property of solid magnetic materials and fluidity of liquids, which makes it an 
attractive material. MF has many unique magneto-optical effects such as linear dichroism, linear birefringence, Faraday rotation and Faraday ellipticity. The magneto-optical properties of MFs imply their various applications in optical and sensing fields, such as magnetic field sensors [1-9], slowing light [10], optical switches [11] and modulators [12]. One of the major applications is magnetic field sensing with optical techniques. On the other hand, due to the advantages of compact structure, high precision and resolution, fiber-optic structures have been widely used in many applications. Therefore, the fiber-based magnetic field sensing using MF as the sensitive material is getting continuing interest recently [13-15].

Multimode-singlemode-multimode (MSM) fiber structures have been successfully utilized to sense temperature [16] and bend [17]. In this work, the MSM structure combining with MF is employed for magnetic field sensing, which has the advantages of low cost and ease of fabrication. In other reported works, considerably complicated techniques are usually involved, such as tapering [3,6], corroding [5], core-offset fusion splicing [6] and microfabrication [13]. In our work, the simple fusion splicing technique is used. The high-order modes are excited in the singlemode fiber (SMF) cladding and then interfere with the core mode. The effective refractive indices of the involved cladding modes can be influenced by the external environment. Then, the sensing purpose is achieved.

\section{Experiments and Principles}

The proposed MSM structure consisting of a segment of SMF sandwiched between two sections of multimode fibers (MMFs), which is illustrated in Figure 1. The MMFs act as the lead-in and lead-out fibers, respectively. The core and cladding diameters of the MMF are 105 and $125 \mu \mathrm{m}$, respectively. The length of SMF is $30 \mathrm{~mm}$. The as-fabricated MSM structure is put into the capillary filled with MF. The inner diameter of the capillary is about $2 \mathrm{~mm}$. Both ends of the capillary are sealed to avoid MFs leaking or evaporating. In our experiments, the water-based $\mathrm{Fe}_{3} \mathrm{O}_{4} \mathrm{MFs}$ with saturation magnetization of $\sim 20 \mathrm{mT}$ and density of $1.18 \times 10^{3} \mathrm{~kg} / \mathrm{m}^{3}$ are provided by Beijing Sunrise Ferrofluid Technological Co., Ltd. The diameter of the magnetic nanoparticles within the MFs is around $10 \mathrm{~nm}$.

Figure 1. Schematic of the multimode-singlemode-multimode (MSM) structure. The low panel shows the experimental structure.

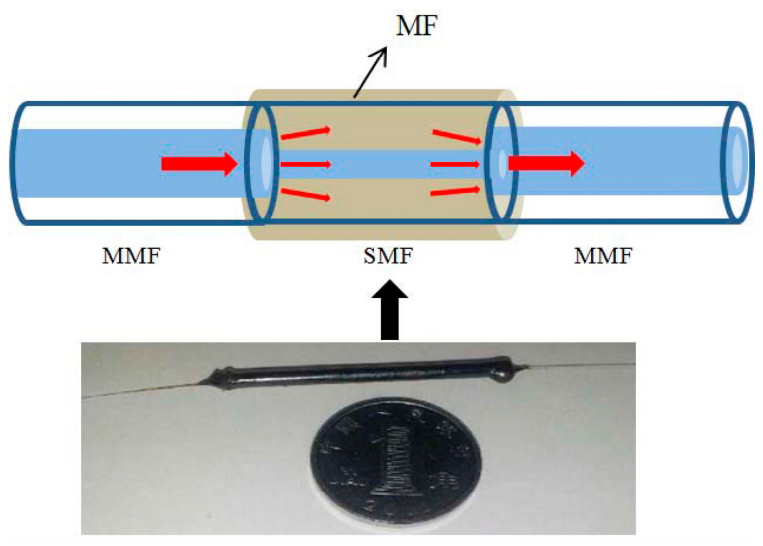

The experimental setup for investigating the magnetic field sensing properties of the MSM structure is schematically shown in Figure 2. The strength of the magnetic field can be tuned by adjusting the 
supply current. The light from the broadband light source (BBS) is coupled into the lead-in MMF and then approaches the SMF. Due to the large mode field mismatch, the lead-in MMF plays the role of mode coupler to couple the fundamental core mode of the lead-in MMF into the core and cladding modes of the sensing SMF. The interference between the core and cladding modes occurs in the lead-out MMF and is transmitted into the optical spectrometer analyzer (OSA). The transmission spectrum can be analyzed by using the two-mode interference model for simplification [18,19]

$$
\operatorname{Iout}(\lambda)=\operatorname{Icore}(\lambda)+\operatorname{Iclad}(\lambda)+2 \cos (2 \pi \Delta n L / \lambda)
$$

where $\mathrm{I}_{\text {core }}$ and $\mathrm{I}_{\text {clad }}$ are the intensities of the fundamental mode and high-order cladding modes, respectively. $\Delta \mathrm{n}$ is the effective refractive index difference between the core mode and cladding mode. $L$ is the effective length of the sensing section, which equals the length of SMF sandwiched between the MMFs in our experiments. According to Equation (1), the interference valley wavelength $\lambda_{\mathrm{m}}$ can be described as

$$
\lambda_{\mathrm{m}}=2 \Delta \mathrm{nL} /(2 \mathrm{~m}+1)
$$

where $\mathrm{m}$ is the interference order.

Figure 2. Experimental setup for investigating the magnetic field sensing properties of the MSM structure.

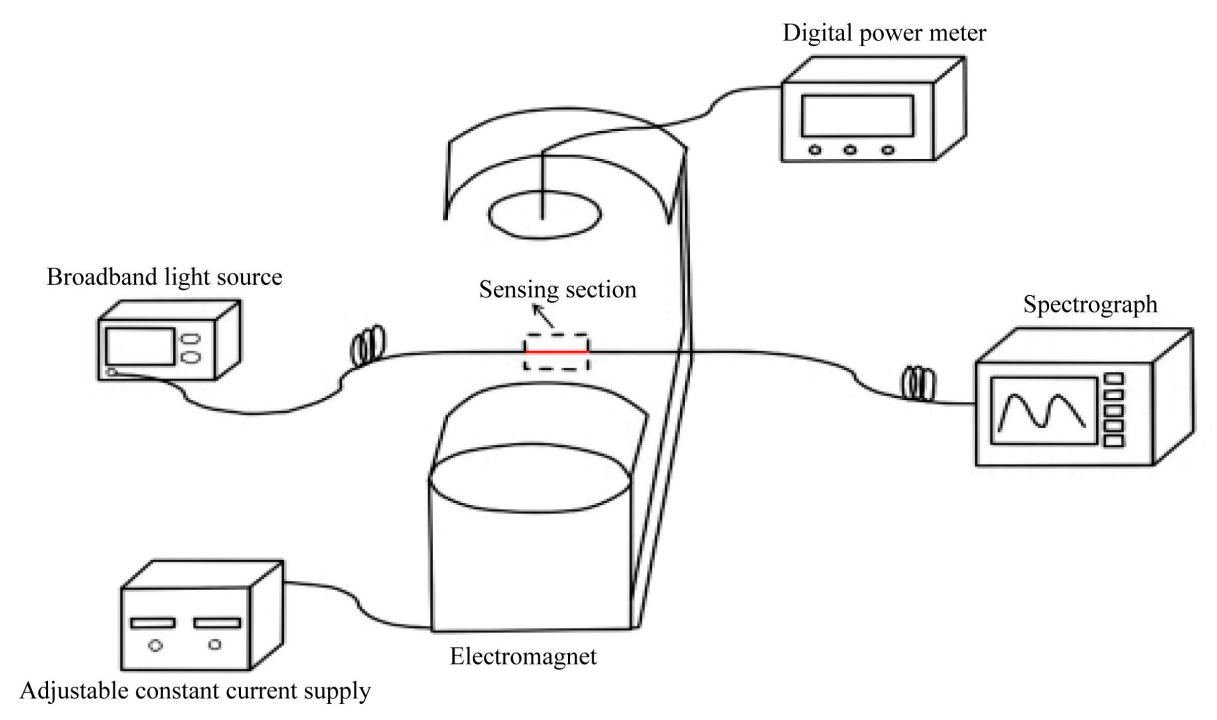

The effective refractive index of the core mode is unaffected but the cladding modes will be influenced by the environmental refractive index change. So the effective refractive index difference between the core and cladding modes will vary with the environmental refractive index, which will result in the shift of transmission spectrum according to Equation (1). Besides, the cladding mode energy leakage will vary with the mode effective refractive index difference. Therefore, the transmission loss of the sensing structure will change with the environmental refractive index as well. It is well known that MFs have unique properties of magnetic-field-dependent refractive index [20-22]. When MFs are used as the cladding of the sensing section, the interference valley wavelength and transmission loss may change with the magnetic field, which can be employed for magnetic field sensing. 


\section{Results and Discussion}

The principle of the magnetic field sensing of the proposed structure is based on the refractive index sensing in nature. Hence, the sensing properties of the MSM structure in a wide range of refractive index variation will be characterized first. The magnetic field sensing properties are then investigated using a specific MF as the cladding of the MSM structure. To obtain various liquids with different refractive indices, the glycerinum liquids with different concentrations are prepared. The refractive indices of the as-prepared glycerinum liquids are listed in Table 1.

Table 1. Refractive index of glycerinum liquids with different concentrations.

\begin{tabular}{cccccccc}
\hline \multirow{2}{*}{ Liquids } & \multirow{2}{*}{ Water } & \multicolumn{6}{c}{ Glycerinum Liquids with Different Concentrations } \\
\cline { 3 - 8 } & & $\mathbf{1 0 \%}$ & $\mathbf{2 0 \%}$ & $\mathbf{3 0 \%}$ & $\mathbf{4 0 \%}$ & $\mathbf{5 0 \%}$ & $\mathbf{6 0 \%}$ \\
\hline RI & 1.3300 & 1.3448 & 1.3575 & 1.3707 & 1.3841 & 1.3909 & 1.4123 \\
\hline
\end{tabular}

The typical transmission spectra of the sensing structure surrounded with glycerinum liquids of different concentrations are shown in Figure 3. Two distinct interference valleys are observed in the wavelength range of 1560 to $1610 \mathrm{~nm}$. The interference valley around $1565 \mathrm{~nm}$ is referred as dip 1 and that around $1595 \mathrm{~nm}$ is referred as dip 2 . Figure 3 indicates that both of the interference valleys shift to long wavelength side with the refractive index increase of the surrounded liquids. In our experiment, the refractive index of SMF is constant and larger than those of the liquids surrounding the SMF. So, most of the mode energy is confined inside the SMF and then the effective refractive indices of the cladding modes are mainly related with the variation of mode energy inside the SMF. With the refractive index increase of surrounding liquid, the cladding mode energy inside the fiber will decrease, which will result in the decrease of effective refractive index of the cladding mode. Therefore, $\Delta \mathrm{n}$ will increase with the refractive index increase of surrounding liquid. This will lead to the red-shift of the wavelength valley according to Equation (2). In addition, the depth of the interference valley decreases with the refractive index increase. It may be assigned to the relatively large field outside the fiber for the higher refractive index case and then the loss due to the external environment increases and the interference effect is weakened.

Figure 3 indicates that the wavelength and intensity of the interference dip are sensitive to the refractive index of the environmental liquids. It is well known that the refractive index of MF can be influenced by a magnetic field. Hence, magnetic field sensing may be realized when using MF as the cladding of the MSM structure.

The transmission spectra of the sensing structure at magnetic field strength ranging from 0 to $16 \mathrm{mT}$ are described in Figure 4. The corresponding intensities of the interference valleys as functions of magnetic field are plotted in Figure 5. From Figures 4 and 5, we can find that the depths of the interference valleys decrease with the magnetic field monotonously. With the magnetic field increases, the interference valleys become shallower and shallower. Then, the tendency of saturation is achieved. These are assigned to the unique properties of magnetic-field-dependent refractive index and loss of MF. The maximum magnetic field sensing sensitivities of $0.4821 \mathrm{~dB} / \mathrm{mT}$ and $0.5742 \mathrm{~dB} / \mathrm{mT}$ can be obtained for dips 1 and 2, respectively. The sensitivities for dips 1 and 2 are different. The possible reason is that the interference between different modes results in dips 1 and 2, respectively. Different modes have 
different sensitivities to the external surrounding [23]. The proposed sensing structure is immersed in MF. The refractive index of MF would be changed with the change of external magnetic field. For the wavelength interrogation, the sensitivity of the proposed structure is higher $(215 \mathrm{pm} / \mathrm{mT})$ than that of [5] $(-168.6 \mathrm{pm} / \mathrm{mT})$. For the intensity interrogation, the sensitivity of the proposed structure is higher $(0.5742 \mathrm{~dB} / \mathrm{mT})$ than the highest sensitivity of [6] $(0.3407 \mathrm{~dB} / \mathrm{mT})$. Compared to [8], the proposed structure is simple and cost-effective. Zheng et al. [8] used a tilted-fiber Bragg grating cascaded with a chirped-fiber Bragg grating.

Figure 3. Transmission spectra of the proposed sensing structure surrounded with glycerinum liquids of different concentrations.

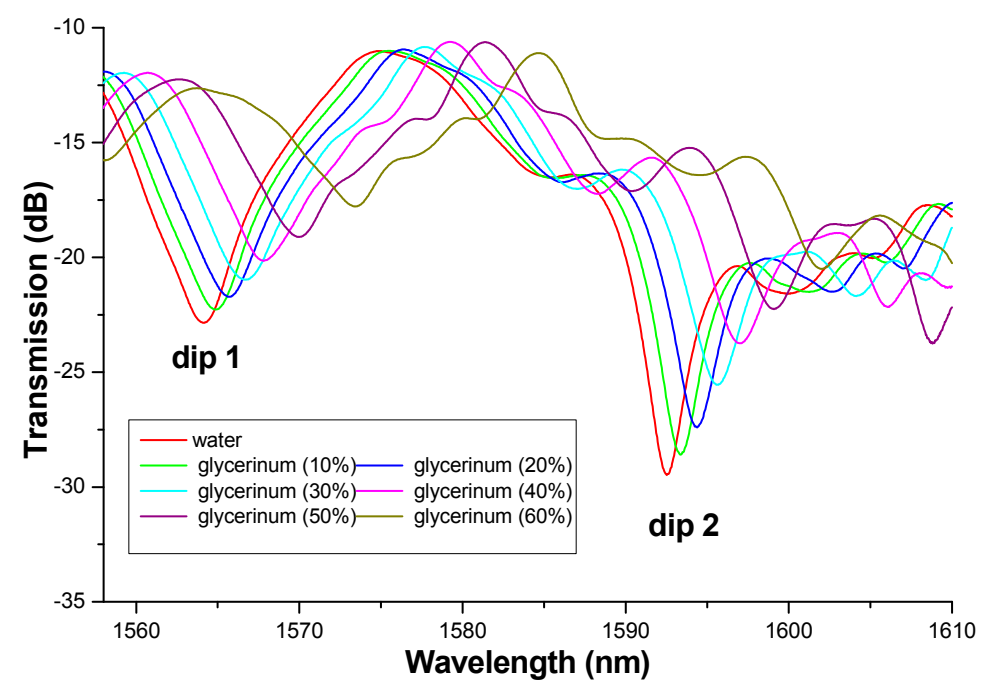

Figure 4. Transmission spectra of the proposed sensing structure at magnetic field strength ranging from 0 to $16 \mathrm{mT}$.

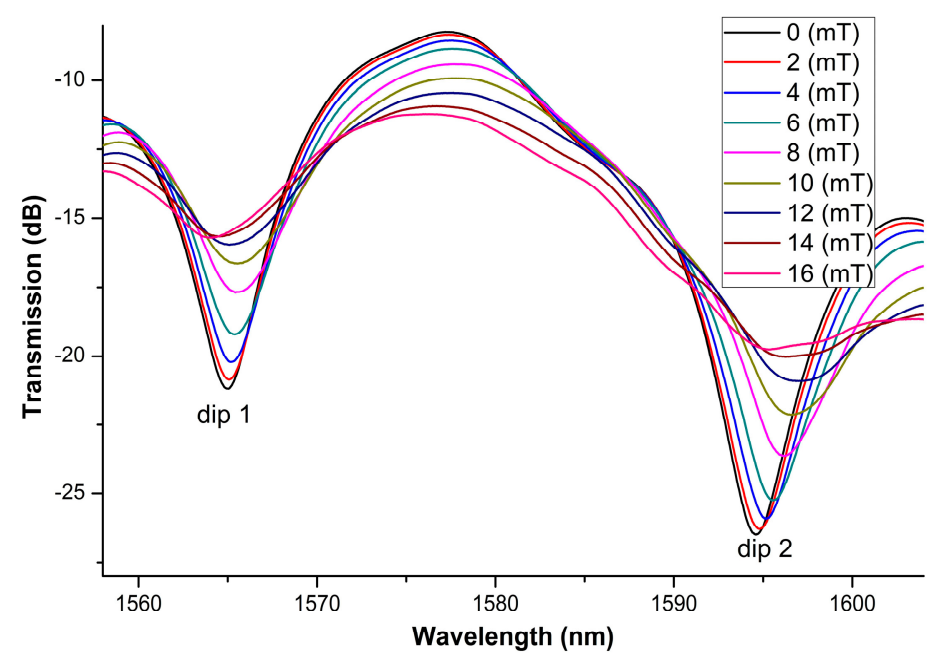

The dip wavelength as a function of external magnetic field is shown in Figure 6, which displays a linear dependence at low field regime. Figures 5 and 6 indicate that a good linear relationship is found at moderate magnetic field strength. These are due to the unique magneto-optical properties of MFs. Therefore, the linear fitting is only made at a moderate magnetic field region. Besides, linear relationship 
is favorable for practical sensing application from the point of interrogation. The linear fitting method has also been employed by other authors (for example, see [5,6]). The sensitivities are obtained to be $60.5 \mathrm{pm} / \mathrm{mT}$ and $215 \mathrm{pm} / \mathrm{mT}$ for dips 1 and 2 , respectively.

Figure 5. Intensities of dip 1 and dip 2 as functions of magnetic field.

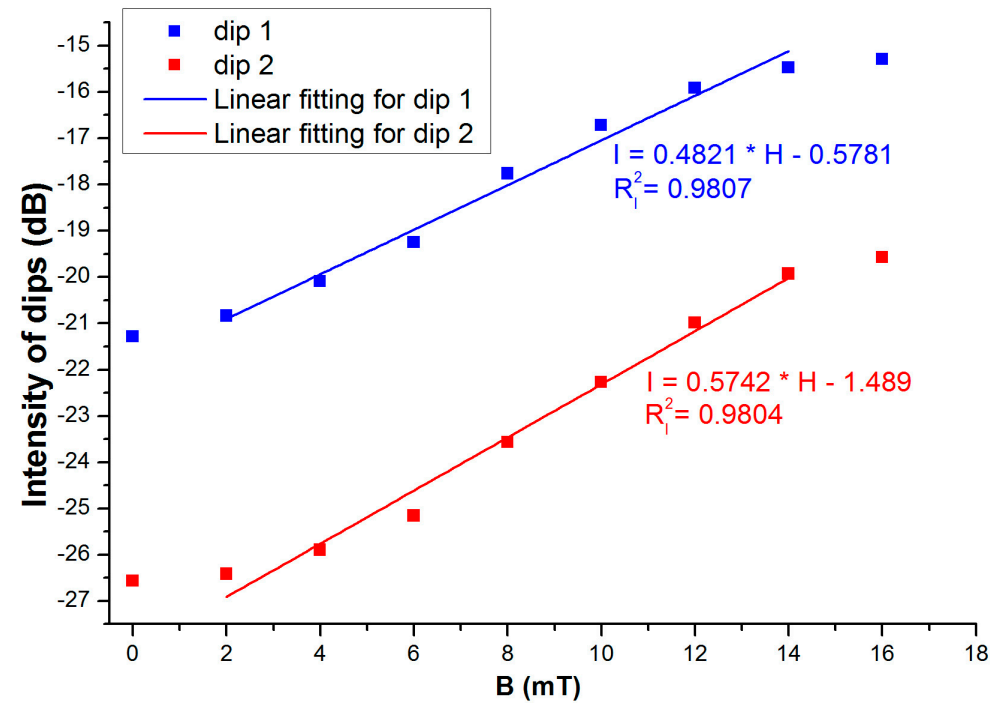

Figure 6. Wavelengths of dips 1 and 2 change with magnetic field.

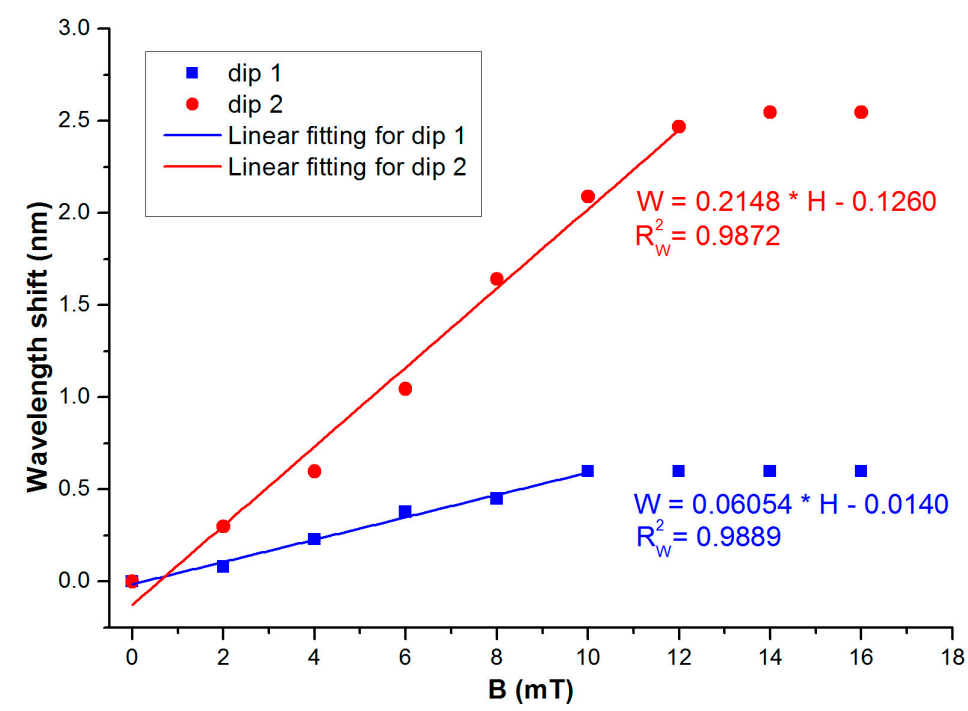

To study the effect of temperature on the sensing performance of the proposed MSM structure, the experiments are conducted at various temperatures. The corresponding results are described in Figures 7 and 8 . Figures 7 and 8 show that the dip wavelength blue-shifts with temperature. The refractive index of MF decreases with temperature. Consequently, $\Delta \mathrm{n}$ will decrease with temperature, which will result in the blue-shift of the wavelength valley. From Figure 8, the maximum sensitivity of temperature effect is obtained to be $9.93 \mathrm{pm} /{ }^{\circ} \mathrm{C}$ (for dip 2). So for some applications (e.g., temperature variation is remarkable or high precision magnetic field measurement), temperature calibration is needed. 
Figure 7. Transmission spectra of the proposed sensing structure at different temperatures.

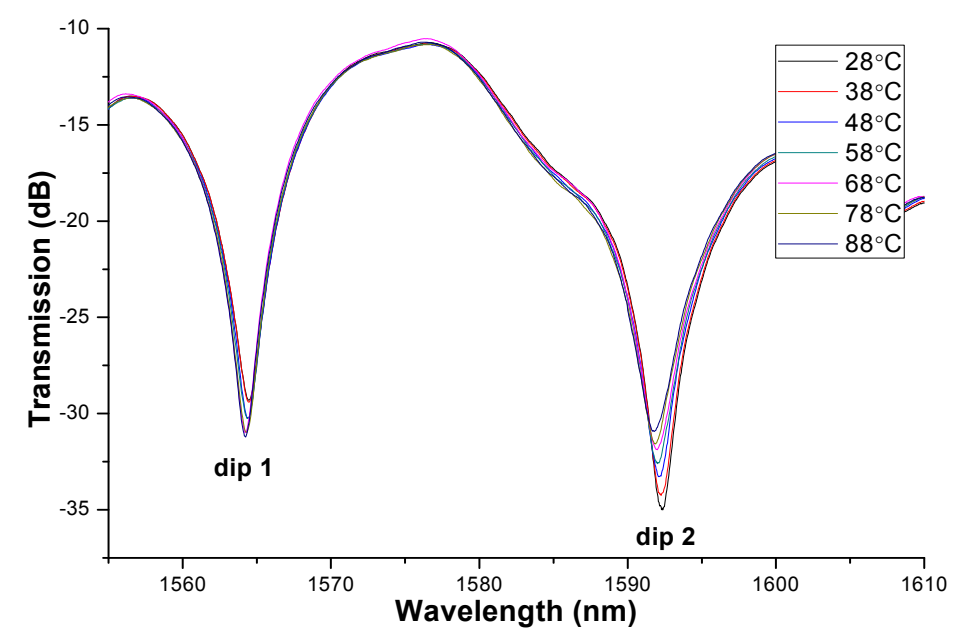

Figure 8. Wavelength of dips 1 and 2 change with temperature.

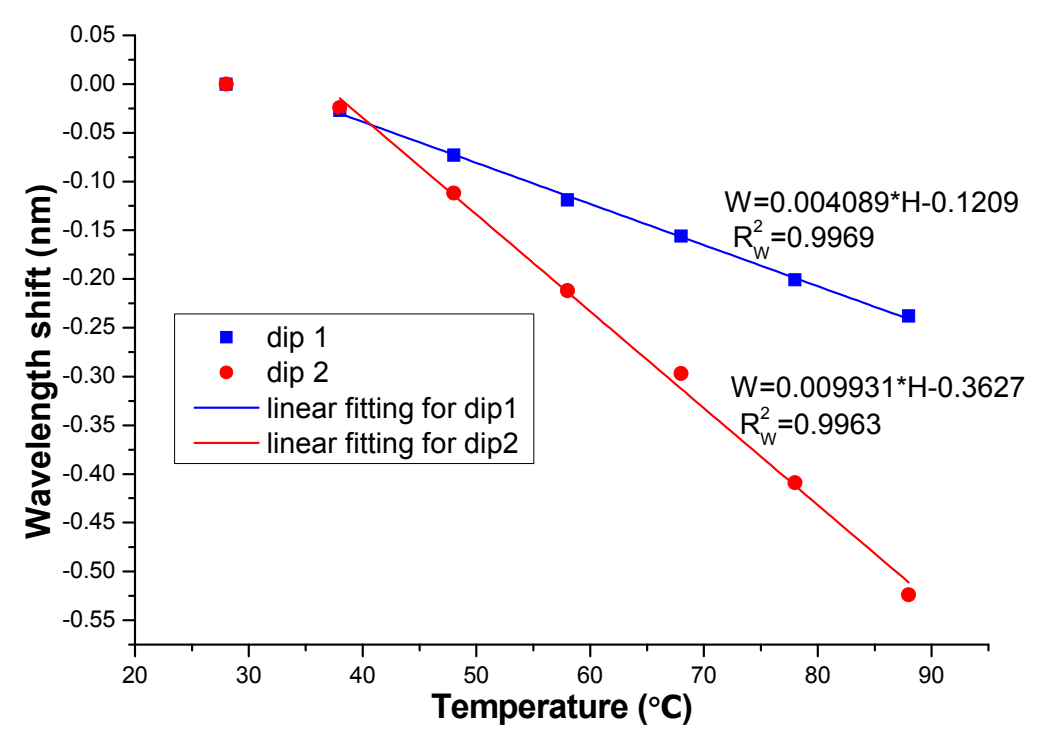

\section{Conclusions}

In summary, a kind of magnetic field sensor based on MF-clad SMF sandwiched between two pieces of MMFs is proposed. It was found that the depth of the interference valley varies with the magnetic field strength. Meanwhile, the interference valley wavelengths change with the external magnetic field monotonously. The corresponding sensitivities are obtained to be $0.4821 \mathrm{~dB} / \mathrm{mT}$ and $60.5 \mathrm{pm} / \mathrm{mT}$ for the wavelength dip around $1565 \mathrm{~nm}$. For the wavelength dip around $1595 \mathrm{~nm}$, the sensitivities are $0.5742 \mathrm{~dB} / \mathrm{mT}$ and $215 \mathrm{pm} / \mathrm{mT}$. The temperature effect of the sensing structure is also investigated. The temperature sensitivity can reach $9.93 \mathrm{pm} /{ }^{\circ} \mathrm{C}$. The proposed sensing structure has the advantages of low-cost and compactness.

\section{Acknowledgments}

This research is supported by the Shanghai Natural Science Fund (No. 13ZR1427400) and the Hujiang Foundation of China (B14004). 


\section{Author Contributions}

The experimental measurement and data calculation of this study were mainly done by the first author, JT. SD and LL contributed to experimental work and gave JT some help. SP supervised the study. This article was written by JT and revised by SP. All authors read and approved the final manuscript.

\section{Conflicts of Interest}

The authors declare no conflict of interest.

\section{References}

1. Dong, S.; Pu, S.; Huang, J. Magnetic field sensing based on magneto-volume variation of magnetic fluids investigated by air-gap Fabry-Pérot fiber interferometers. Appl. Phys. Lett. 2013, 103, doi:10.1063/1.4821104.

2. Miao, Y.; Wu, J.; Lin, W.; Zhang, K.; Yuan, Y.; Song, B.; Zhang, H.; Liu, B.; Yao, J. Magnetic field tunability of optical microfiber taper integrated with ferrofluid. Opt. Express 2013, 21, 29914-29920.

3. Deng, M.; Liu, D.; Li, D. Magnetic field sensor based on asymmetric optical fiber taper and magnetic fluid. Sens. Actuators A Phys. 2014, 211, 55-59.

4. Ji, H.; Pu, S.; Wang, X.; Yu, G. Magnetic field sensing based on V-shaped groove filled with magnetic fluids. Appl. Opt. 2012, 51, 1010-1020.

5. Wang, H.; Pu, S.; Wang, N.; Dong, S.; Huang, J. Magnetic field sensing based on singlemode-multimode-singlemode fiber structures using magnetic fluids as cladding. Opt. Lett. 2013, 38, 3765-3768.

6. Wu, J.; Miao, Y.; Lin, W.; Song, B.; Zhang, K.; Zhang, H.; Liu, B.; Yao, J. Magnetic-field sensor based on core-offset tapered optical fiber and magnetic fluid. J. Opt. 2014, 16, doi:10.1088/ 2040-8978/16/7/075705.

7. Candiani, A.; Argyros, A.; Leon-Saval, S.G.; Lwin, R.; Selleri, S.; Pissadakis, S. A loss-based, magnetic field sensor implemented in a ferrofluid infiltrated microstructure polymer optical fiber. Appl. Phys. Lett. 2014, 104, doi:10.1063/1.4869129 .

8. Zheng, J.; Dong, X.; Zu, P.; Ji, J.; Su, H.; Shum, P. Intensity-modulated magnetic field sensor based on magnetic fluid and optical fiber gratings. Appl. Phys. Lett. 2013, 103, doi:10.1063/1.4828562.

9. Yang, D.; Du, L.; Xu, Z.; Jiang, Y.; Xu, J.; Wang, M.; Bai, Y.; Wang, H. Magnetic field sensing based on tilted fiber bragg grating coated with nanoparticle magnetic fluid. Appl. Phys. Lett. 2014, 104, doi:10.1063/1.4864649.

10. Pu, S.; Dong, S.; Huang, J. Tunable slow light based on magnetic-fluid infiltrated photonic crystal waveguides. J. Opt. 2014, 16, doi:10.1088/2040-8978/16/4/045102.

11. Kemkar, S.D.; Mahajan, H.S.; Vaidya, M. Ferro fluid based optical fiber switch. In Proceedings of the 56th Dae Solid State Physics Symposium 2011, Tamilnadu, India, 19-23 December 2011; doi:10.1063/1.4710108. 
12. Chieh, J.J.; Yang, S.Y.; Horng, H.E.; Hong, C.-C.; Yang, H.C. Magnetic-fluid optical-fiber modulators via magnetic modulation. Appl. Phys. Lett. 2007, 90, doi:10.1063/1.2716365.

13. Zu, P.; Chan, C.C.; Koh, G.W.; Lew, W.S.; Jin, Y.; Liew, H.F.; Wong, W.C.; Dong, X. Enhancement of the sensitivity of magneto-optical fiber sensor by magnifying the birefringence of magnetic fluid film with Loyt-Sagnac interferometer. Sens. Actuators B Chem. 2014, 191, 19-23.

14. Miao, Y.; Liu, B.; Zhang, K.; Zhang, H.; Wang, R.; Liu, Y.; Yao, J. Magneto-optical tunability of magnetic fluid infiltrated microstructured optical fiber. Opt. Laser Technol. 2013, 48, 280-284.

15. Homa, D.; Pickrell, G. Magnetic sensing with ferrofluid and fiber optic connectors. Sensors 2014, 14, 3891-3896.

16. Nguyen, L.V.; Hwang, D.; Moon, S.; Moon, D.S.; Chung, Y. High temperature fiber sensor with high sensitivity based on core diameter mismatch. Opt. Express 2008, 16, 11369-11375.

17. Sun, A.; Wu, Z.; Wan, C.; Yang, C. All-fiber optic acoustic sensor based on multimode-single mode-multimode structure. Optik 2012, 123, 1138-1139.

18. Deng, M.; Sun, X.; Han, M.; Li, D. Compact magnetic field sensor based on optical microfiber Michelson interferometer and $\mathrm{Fe}_{3} \mathrm{O}_{4}$ nanofluid. Appl. Opt. 2013, 52, 734-741.

19. Jha, R.; Villatoro, J.; Badenes, G.; Pruneri, V. Refractometry based on a photonic crystal fiber interferometer. Opt. Lett. 2009, 34, 617-619.

20. Zhao, Y.; Wu, D.; Lv, R.; Ying, Y. Tunable characteristics and mechanism analysis of the magnetic fluid refractive index with applied magnetic field. IEEE Trans. Magn. 2014, 50, doi:10.1109/TMAG.2014.2310710.

21. Yang, S.Y.; Chieh, J.J.; Horng, H.E.; Hong, C.-Y.; Yang, H.C. Origin and applications of magnetically tunable refractive index of magnetic fluid films. Appl. Phys. Lett. 2004, 84, 5204-5206.

22. Hong, C.-Y.; Yang, S.Y.; Horng, H.E.; Yang, H.C. Control parameters for the tunable refractive index of magnetic fluid films. J. Appl. Phys. 2003, 94, 3849-3852.

23. Shu, X.; Zhang, L.; Bennion, I. Sensitivity characteristics of long-period fiber gratings. IEEE J. Lightwave Technol. 2002, 20, 255-266.

(C) 2014 by the authors; licensee MDPI, Basel, Switzerland. This article is an open access article distributed under the terms and conditions of the Creative Commons Attribution license (http://creativecommons.org/licenses/by/4.0/). 